Z Herz- Thorax- Gefäßchir 2019 · 33:3-5

https://doi.org/10.1007/s00398-018-0290-2

(c) Springer Medizin Verlag GmbH, ein Teil von Springer Nature 2019

CrossMark

\author{
H. E. Ulmer \\ Heidelberg, Deutschland
}

\title{
Aldo Ricardo Castaneda
}

Guatemala City, sein Medizinstudium. In seinem 2. Studienjahr lernte er eine junge Frau kennen, die den Krieg ebenfalls in Europa verbracht hatte. 1956, noch während seines Studiums, heiratete er Arcely Rey-Rosa. Die beiden leben seitdem länger als 60 Jahre zusammen und sind Eltern zweier Töchter und eines Sohns.

1958 schloss er sein Medizinstudium als Bester seines Jahrgangs ab, mit einer Arbeit unter dem Titel: „Chirurgie am offenen Herzen: Eine experimentelle Studie“. Sie befasste sich mit tierexperimentellen Versuchen zur extrakorporalen Zirkulation und brachte ihm die erste seiner später zahlreichen Auszeichnungen ein, den Rufino Barrios-Preis der Universität San Carlos. Das weitere Interesse war nicht einfach zu realisieren: eine chirurgische Ausbildung in den USA? Träume in Guatemala?

Der junge Medizinstudent war anlässlich wissenschaftlicher Kongresse an seiner Universität gelegentlich als Simultandolmetscher eingesetzt gewesen. Einmal hatte er dabei den Vortrag eines norwegischen Kinderpsychiaters aus Minneapolis zu übersetzen. Einen unerwartet in den Vortrag eingebauten Scherz in norwegischer Sprache konnte er aber nicht verstehen und bat daher sein Publikum durch sein Übersetzermikrofon, dennoch zu lachen, was auch spontan befolgt wurde. Aldo war für den Wissenschaftler aus Minnesota „... ein junges Genie aus Guatemala“. Es wird berichtet, dass dieses Ereignis letztlich die Entscheidung der University of Minnesota in Minneapolis herbeigeführt haben soll, den Antrag eines ansonsten unbekannten jungen $\mathrm{Me}$ diziners aus Mittelamerika auf ein Stipendium positiv zu bescheiden. Damit landete Aldo Castaneda 1959 in Minneapolis, direkt im Zentrum der „norwegischen Herztruppe“ von Olaf Wan- gensteen, Walton Lillehei, Richard Varco und anderen. Der wissenschaftliche Weg in die gerade beginnende Chirurgie des Herzens war damit vorgezeichnet.

Es folgten 1963 der Master-Abschluss in Biochemie und 1964 der PhD in Physiologie und experimenteller Chirurgie, bei gleichzeitigem Abschluss der Ausbildung in "General Surgery“. In den nachfolgenden Jahren entwickelten sich in diesem anregenden Umfeld das Interesse und die besonderen praktischen Fähigkeiten von Aldo Castaneda auf dem gerade in Entstehung begriffenen Gebiet der Chirurgie angeborener Herzfehler. Minneapolis nahm hierbei rasch eine der führenden Positionen in den USA ein und zog damit zahlreiche, am Herzen interessierte Chirurgen aus aller Welt als Besucher an. 1970, d.h. 12 Jahre nach dem Abschluss seines Studiums in Guatemala, wurde Aldo Castaneda an der Universität von Minnesota zum „Full Professor of Surgery“ ernannt.

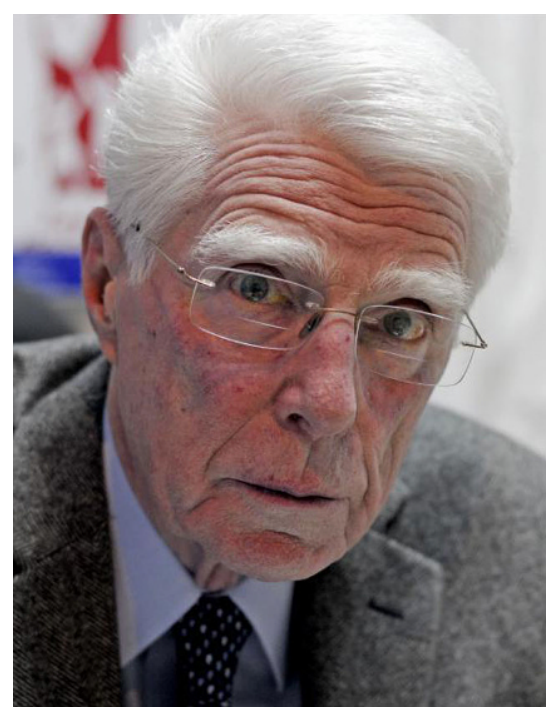

Abb. 1 A Aldo Ricardo Castaneda (geb. 1930). ( ) Alle Rechte vorbehalten 


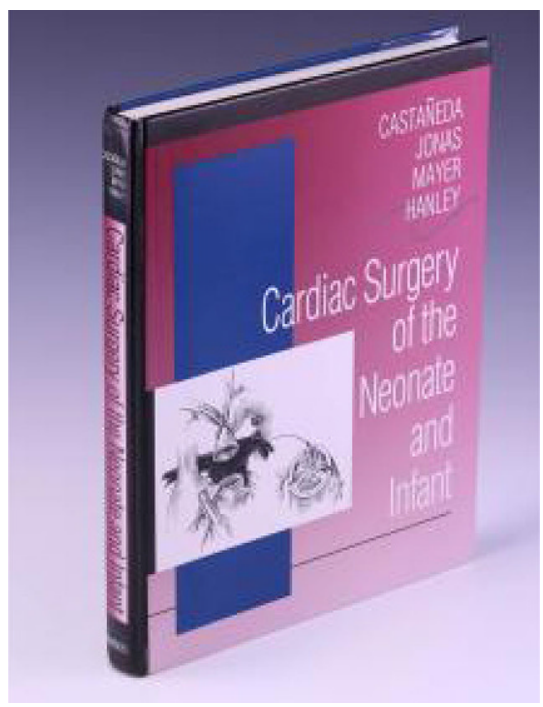

Abb. $2 \Delta$ Aldo R. Castaneda et al. „Cardiac Surgery of the Neonate and Infant". (1st edition 1994, Saunders, Philadelphia 1994). @ Alle Rechte vorbehalten

1972 legte Robert Gross, der 1938 mit einer Duktusligatur die erste geplante Operation eines angeborenen Herz-/ Gefäßfehlers durchgeführt hatte, die Leitung der „Cardiovascular Surgery“ im Boston Children's Hospital nieder. Aus einer großen Gruppe renommierter internationaler Kandidaten wurde Aldo Castaneda als sein Nachfolger ausgewählt und trat im Oktober 1972 das Amt des „Professor of Surgery“ an der Harvard Medical School an, verbunden mit der Position des "Cardiac Surgeon in Chief“. 1975, nach dem vollständigen Ausscheiden von Robert Gross, wurde er auch dessen Nachfolger als der zweite Inhaber der William-E.-Ladd-Professur „of Child Surgery“ an der Harvard Medical School. Alle diese Positionen übte er bis zu seinem eigenen Ausscheiden im Jahr 1994 in Boston aus.

Die Übernahme der Herzchirurgie des Children's Hospital durch Aldo Castaneda bedeutete für Boston als Kinderherzzentrum einen Schritt in ein anderes Zeitalter, da Gross nach seiner ersten Großtat der weiteren Entwicklung der Chirurgie angeborener Herzfehler kein allzu großes Interesse mehr entgegengebracht hatte. Organisatorisch bedeutete dies die Aufteilung der Klinik in zwar eigenverantwortliche Bereiche (1972!), jedoch mit der absoluten Verpflichtung zur Arbeit als Team. Regelmäßige gemeinsa-

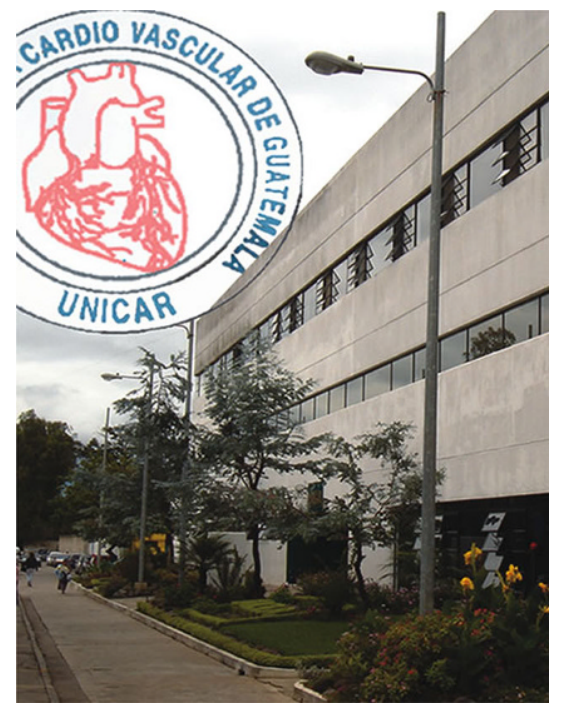

Abb. 3 ^ National Heart Institute, Guatemala City. ( ) Alle Rechte vorbehalten

me klinische Konferenzen und ein detailliertes zertifiziertes Trainingsprogramm für die Kinderherzchirurgie wurden zu gerühmten und oft kopierten Selbstverständlichkeiten. Die wichtigste Entwicklung war jedoch der Weg der Chirurgie angeborener Herzfehler in die Säuglings- und Neugeborenenperiode unter der Vorstellung, dadurch Sekundärschäden am Herzen und an anderen Organen des Körpers zu vermeiden. Dieses Konzept führte nahezu erwartungsgemäß zu einer heftig umstrittenen Diskussion in der übrigen herzchirurgischen Welt, wobei die in Boston erzielten Ergebnisse dieses Vorgehen jedoch im Laufe der Zeit zum angestrebten Standard an den großen Zentren der Welt machten.

Aldo Castaneda und der von ihm geförderte William Norwood, der ihm bereits aus Minneapolis gefolgt war, stellten den Kern dieser sich in Boston entwickelnden Elitemannschaft dar und wurden gelegentlich auch als das Yin und Yang dieser neuen Kinderherzchirurgie bezeichnet. Am 2. Januar 1983 führten sie gemeinsam die erste sog. arterielle Switch-Operation nach dem Prinzip von Jatene bei einem 11 Tage alten Neugeborenen mit einer Transposition der großen Arterien erfolgreich durch. Noch im selben Jahr publizierte Norwood die erste erfolgreiche Palliation bei einem Neugeborenen mit einem hypoplastischen
Linksherzsyndrom, dem letzten bis dahin als nicht chirurgisch behandelbar angesehenen angeborenen Herzfehler. Trotz dieses aus ethischen Gründen international erneut heftig umstrittenen Vorgehens wurde Aldo Castaneda im folgenden Jahr 1984 mit dem Lifetime Achievement Award des American College of Cardiology ausgezeichnet. Sein nachhaltiges und letzten Endes erfolgreiches Eintreten für die Frühkorrektur gerade komplexer angeborener Herzfehler wird heute als sein bedeutendster Beitrag zur Entwicklung der Kinderherzmedizin allgemein angesehen und drückt sich aus in der „Castaneda doctrine“: „Operate as soon postnatally as the patient needs it. Whenever possible do corrective surgery, not palliative surgery“ (• Abb. 2).

1994, nach mehr als 24 Jahren chirurgischer und akademischer Tätigkeit, nahm Aldo Castaneda seinen Abschied vom Boston Children's Hospital. Noch im selben Jahr begann er, zusammen mit William Norwood, in einer großen privaten Klinik in Genolier in der Schweiz eine an den chirurgischen Fähigkeiten der beiden orientierte Kinderherzchirurgie einzurichten, die innerhalb der nächsten drei Jahre rasch einen ausgezeichneten Ruf erwarb. Aus akuten gesundheitlichen Gründen musste er jedoch diese erfolgreiche Unternehmung im September 1997 beenden und kehrte nach Guatemala zurück.

Die Ruhezeit hielt jedoch nicht lange vor. Nach seinen eigenen Worten wollte er „... dorthin etwas zurückgeben, wo er seine Wurzeln fühlte." Er sah seine Aufgabe darin, jetzt im 67. Lebensjahr, in Guatemala City das erste und einzige Zentrum in Zentralamerika für Kinder mit angeborenen Herzfehlern zu errichten. Sein Ziel war es, nicht nur die Herzoperationen vor Ort durchzuführen, sondern ein vollständiges Programm zur Ausbildung von Herzchirurgen und Kinderkardiologen $\mathrm{zu}$ etablieren sowie mit angeschlossenen Forschungslabors neue Entwicklungen auf diesem Gebiet nachhaltig zu fördern. Noch 1997 begann die bauliche Vergrößerung des Guatemala Heart Center UNICAR, einer bestehenden Einrichtung für internistische Kardiologie in Guatemala City. 1998 wurde 
als Ergänzung das von der neu gegründeten Aldo Castaneda Foundation finanzierte und nach den modernsten Richtlinien gestaltete Kinderherzzentrum eröffnet (• Abb. 3).

Anfangs war Aldo Castaneda der einzige qualifizierte Operateur und führte alle Operationen selbst und für die Eltern kostenlos durch. Heute umfasst der Stab 3 von ihm selbst ausgebildete zertifizierte Kinderherzchirurgen, 7 Kinderkardiologen, 2 OP, eigene Katheter- und Echolabors sowie eine eigene kardiologische Intensivstation für Kinder. Inzwischen hat sich die Klinik zum Referenzzentrum für Kinderherzchirurgie für den gesamten Bereich der zentralamerikanischen Staaten entwickelt.

Im Mai 2006 wurde er in die Hall of Fame der Association for European Paediatric Cardiology (AEPC) aufgenommen. Im Februar 2014, nach mehr als 5000 Operationen und zunehmend ge- zeichnetvon der Bürde des Alters, hat sich Aldo Castaneda im Alter von 84 Jahren von den operativen Aufgaben zurückgezogen und die Leitung des Castaneda Research Laboratory in seiner Klinik übernommen. Im April 2018 wurde er der 8. Träger des Lifetime Achievement Award der American Association for Thoracic Surgery (AATS).

\section{Korrespondenzadresse}

\section{Prof. Dr. H. E. Ulmer}

Am Aukopf 5, 69118 Heidelberg, Deutschland herbert_ulmer@t-online.de

\section{Springer Medizin}

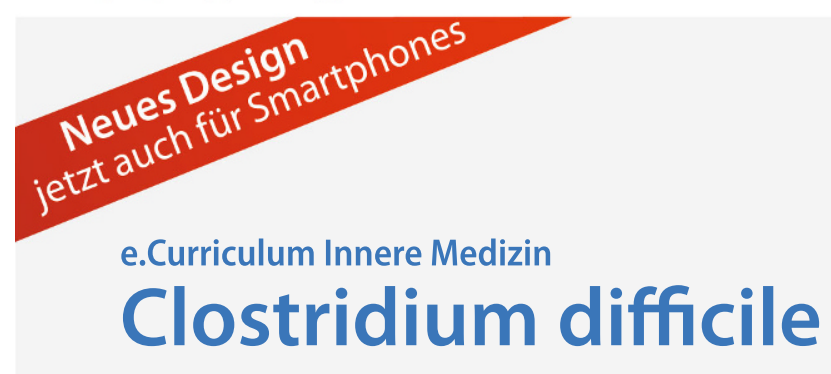

E-Learning

- Nach der aktuellen Leitlinie

- Fallbasiertes E-Learning

- Zertifiziert mit 3 Punkten

- Exklusiv für DGIM-Mitglieder und e.Med-Abonnenten

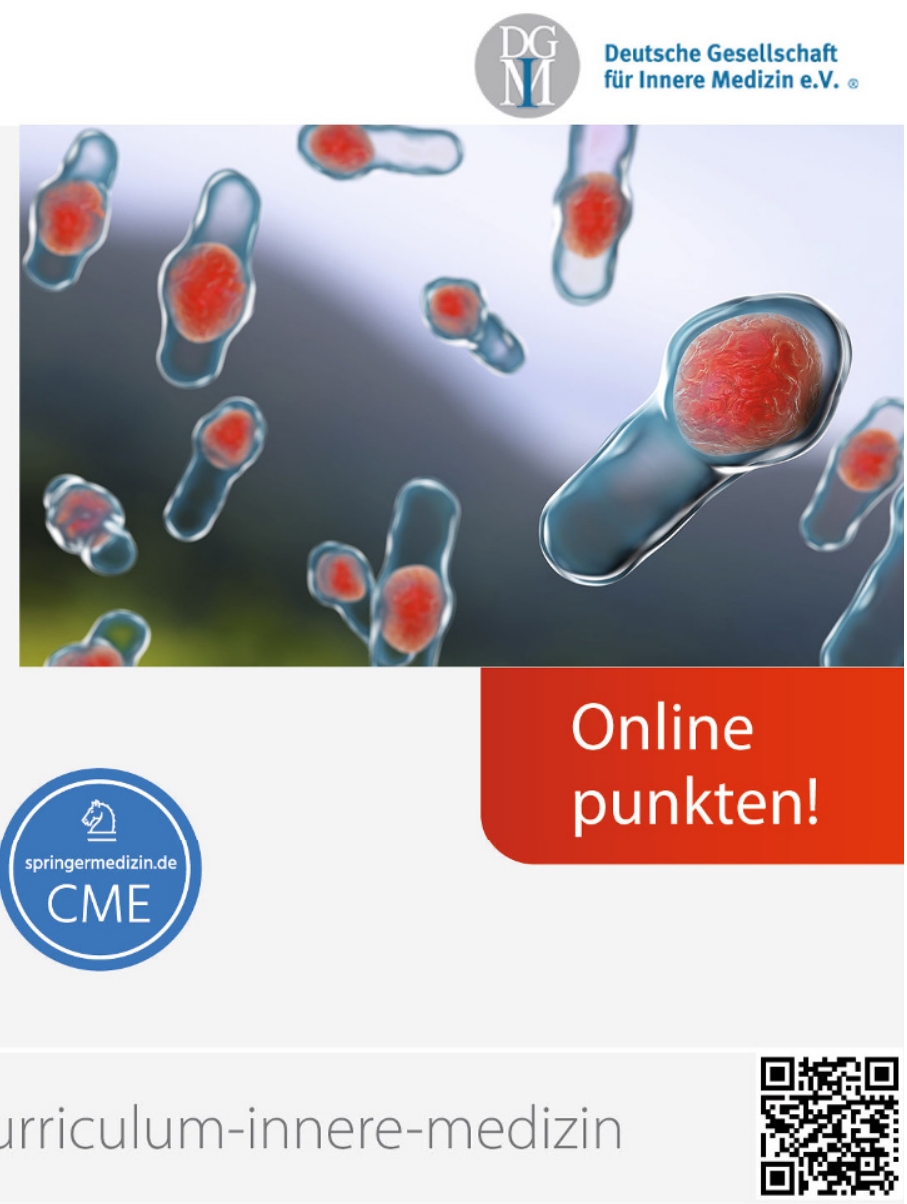

